\title{
A Review and Application of Linear Infinitely Variable Transmission in Automobile
}

\author{
Arun M. Meshram ${ }^{1}$, S. P. Chaphalkar ${ }^{2}$, S. N. Khetre ${ }^{3}$, S. R. Wankhede ${ }^{4}$ \\ ${ }^{1} M E$ Student, Department of Mechanical Engineering, Alard COE\&M, Pune \\ ${ }^{2}$ Head of Department, Department of Automobile Engineering, Pimpri Chinchwad, Polytechnic, Pune, \\ ${ }^{3}$ Assistant professor, Department of Mechanical Engineering, JSPM Rajarshi Shahu COE, Pune \\ ${ }^{4}$ Assistant professor, Department of Mechanical Engineering, Pimpri Chinchwad COE, Pune \\ Savitribai Phule Pune University, Pune, Maharashtra, India.
}

\begin{abstract}
In this paper, the linear Infinitely Variable Transmission in automobile engine use particular drive a Cam and number of follower rotary mounted a carrier plate to generate an auxiliary motion in equal number of planet gear the energy losses conventional transmission are unacceptable in scientific in manner. The variation of transmission including zero transmission ratios in cam and follower. The IVT has number of identical an unit ,each unit contain tree dimensional cam, cam follower, groove wheel, one way clutch(gear planet), though each unit rotational motion is converted in an oscillatory liner motion of variable amplitude and rectified to the rotation motion again. The motion maximum linear transmission ratio to the system depends on geometric design factors. This paper reviews the transmission system with a concept proposal for Infinitely Variable transmission.
\end{abstract}

Keywords: Infinitely Variable Transmission, Cam, Angular Ratio Velocity Theorem, Positive Drive.

\section{Introduction:}

The purpose of this design project was to improve upon an existing Infinitely Variable Transmission (IVT) design for use in Automobile. An IVT is a new design of transmission which utilizes moments produced by rotating offset masses to transfer torque, while varying output speed, from the engine to the output shaft. In an effort to design a reliable and efficient IVT, all components from the existing IVT were analyzed in detail and modified accordingly.

The linear IVT is solving the problem in modern automobile industry to develop new generation in vehicle to sustain. The input and output ratio in traction the linear IVT parameters to sustain the limited Three masses profile and masses the IVT infinity check the depending upon various load condition in gear, clutch, Three masses and Three masses follower. The superior in automobile torque cycle, the linear IVT dissolve input traction to the shaft traction to the output shaft to the transmission solver transmission ratio. The different investigators presents the background and theory related to the IVT, considers alternative designs, and presents the final design. It also outlines improvements for the IVT which will be installed in automobiles.

A Three masses-based Infinitely Variable Transmission system allows a user to vary the speed between input \& output progressively from one positive value to another. Unlike, conventional transmissions the selection of gears is not restricted to a finite number of ratios. The Three masses-based Infinitely Variable Transmission systems can be used in automobile drive applications to improve performance, economy \& functionality.

\section{Objectives Of Research:}

1. Development of kinematic linkage model for kinematic synthesis of torque convertor using CAD, by overlay method.

2. Design and development of Infinitely Variable transmission based on skeleton developed using CAD overlay method

3. Manufacturing of IVT to derive the geared neutral position along with torque and power augmentation with increase in input speed.

\section{Need Of Infinitely Variable Transmission System:}

There are many automobile and mechanical units that under varying circumstances make it desirable to be able to drive at an barely perceptible speed, an inter mediate speed or a high speed. Thus an infinitely variable (or step less speed variation) in which it is possible to get any desirable speed. Some mechanicals hydraulic and electrical devices serve as such step less drives. However the torque Vs speed characteristics of these drives do not match that of step less drives at increased driving torque at low speeds. Hence the need of a step less drive with the following characteristics Step less or infinitely variable speed. 
1. Wide range of speed variation i.e. (Nmax to 2. Shifting form one speed to another should be Nmin).

3. Minimum no of controls for speed changing. shock less

4. Ease of operation.

\section{Relevance Of IVT In Automobile:}

Manual transmissions (MT) are the most common in today's cars. This transmission only allows a limited set of fixed gear ratios. Usually MT disposes of 5 gear ratios, although there are gear boxes with 6 or 7 . The manual transmission of automobile is as shown in figure:

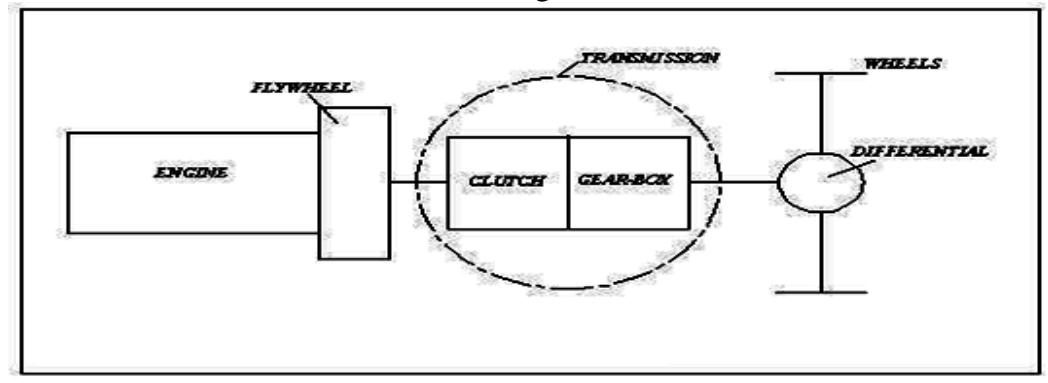

Figure 1. Conventional Automobile transmissions System

Normally the clutch is single plate friction clutch, efficiency $=75 \%$ whereas the gear box is manual transmission gear box with 4 or 5 speeds. ie., Gears $0-1-2-3-4-5$, in order to shift from one gear to another one has to perform three operations ie., De-clutching,Change Gear Gradual engagement thus in order to reach top speed ie., 5th gear we will have to perform 15 operations. This becomes a problem while running vehicle in dense traffic conditions.

- $\quad$ Clutch efficiency $=75 \%$ \& gear box efficiency $=92 \%$ combined transmission efficiency $=67 \%$.

- Gradual engagement requires skill full operation.

- Laborious operation of changing gear every time.

- Low fuel efficiency due to frequent clutch operations.

The Infinitely Variable Transmission (IVT) system is a sophisticated automatic gearbox designed to save on fuel, cut emissions, give a smoother drive and improve performance. Because most motors, whether powered by liquid fuel or electricity, have a narrow RPM range at which they operate at top efficiency, they generally need a transmission to allow for variances in output speed. Most transmissions have a certain number of gears available, meaning there is still significant variation in engine speed before the transmission shifts to the next gear. The purpose of the IVT is to allow the engine to maintain an almost constant speed while the output speed changes to meet the needs of the equipment or process involved The IVT (L-IVT) is a novel transmission design that transmits mechanical power via oscillating torque, rather than traditional speed ratio methods. This unique approach combines the high mechanical efficiency of fixed gear ratio transmission with the high engine efficiency obtained using a Continuously Variable Transmission (CVT) in a lightweight, rugged, and high-torque package. The simple, high-efficiency design of the IVT allows the components to be sized for greater endurance and higher power while remaining smaller than competing designs. The high mechanical efficiency of the L-IVT eliminates hydraulic fluids and costly lubrication and cooling methods resulting in a dramatically higher maintainability and lower operating cost. The small number of parts and the straightforward parts design drive reduced manufacturing costs in both material and labor and increase reliability. The high efficiency design allows the L-IVT to support high load, large vehicle applications previously not supported by existing CVT designs. The L-IVT can scale to support nearly any size application such as Heavy Trucks, Main Battle Tanks or Earth Movers. Unlike conventional transmissions, this infinitely variable transmission (IVT) controls the output torque as opposed to the output speed ratio. Infinitely variable torque, from zero torque to the full capability of torque output, can be produced with no clutching or torque conversion required at the input. The power from the centrifugal forces of rotating eccentric masses is harnessed to create an oscillating torque. One-way clutches convert the oscillating torque to a unidirectional torque. Variable control of the amplitude of the torque results from the change in the centre of gravity of the rotating masses.

\section{Literature Review On IVT:}

A. Dr. N. Arunkumar, (2014) Presented paper on infinitely Variable Transmission Using Four Bar Mechanism [1]. This paper present Most of the continuously variable transmission systems in automobiles now-a-days are non-positive drives. This means that they cannot be used in heavy vehicles that require very high torque to be transmitted. This new type of infinitely variable transmission is aimed at transmitting high torques by making it a positive drive, thus making continuously variable transmission systems to be suitable 
for heavy vehicles. Infinitely variable transmission system and continuously variable transmission system are both the same except that there is an extra zero gear ratio in infinitely variable transmission system. This newly developed transmission system is basically a four bar mechanism with variable crank radius which makes it possible to have continuously variable mechanical advantage. The output lever which oscillates in the four bar mechanism is connected to a ratchet mechanism which turns the output shaft intermittently, two four bar mechanisms with a phase difference of 180 degrees is used to avoid the intermittent rotation of the output shaft. A flywheel is used in the output shaft to reduce the fluctuations in both speed and torque.

B. Derek F. Lahr (2006) Presented paper on the Operation And Kinematic Analysis Of A Novel Cam-Based Infinitely Variable Transmission [2]. In this paper present the operation and analysis of a novel, highly configurable, infinitely variable transmission of the ratcheting drive type is presented. This particular drive uses a cam and a number of cam followers rotatable mounted to a carrier plate to generate an oscillatory motion in an equal number of planet gears. A number of indexing clutches are then used to rectify this motion into a rotational output. A full description of the mechanism, including its components, operation, and kinematic equations are presented. There are a number of inversions of this device, and their characteristics and limitations are discussed. In addition, a method is presented to select the most suitable inversion, gearing, and follower velocity for a given application.

C. Gregory F. Hickman (2013) Presented paper on Kinematic Modeling and Analysis of a Cam Based CVT for a Capstone Design Project Experience [3]. This paper presents a Capstone Design Project at Oakland University, a CAD model of a cam based CVT (Continuously Variable Transmission). The Capstone Design Project at Oakland University covers a broad range of mechanical engineering core disciplines of kinematics, dynamics, material properties and mechanics, machine design, and serves as an integration course before mechanical engineering students' graduate. It takes students through the entire taxonomy of the design process: from searching for ideas, proposal, survey, knowledge, comprehension, application, to analysis, synthesis, and finally evaluation and modification. This project was performed by a group of mechanical engineering senior students, to demonstrate their engineering training and capabilities to search for ideas, to formulate a proposal and modify it to get the approval from the department curriculum committee, to conduct literature survey, to understand the descriptions of a new mechanism, to design and construct a virtual prototype in the computer environment, and finally to propose modifications to improve the original design. This project is to investigate a novel cam based CVT which was proposed in US patent \# 4,603,240, which has a cam input to drive an angle dependent, clutch actuated output shaft. This patent was published 15 years ago, but unfortunately there was no detailed design or analysis ever presented. Based on this patented CVT, a CAD (Computer Aided Design) model utilizing three dimensional CAD software was conducted, creating a visualization and analysis model to ascertain system performance and feasibility. This paper describes the mechanism designed and created, limitation of the modeling software and the approach utilized to overcome these limitations. The resultant motion is then analyzed to ascertain the performance and determine the viability of the design concept. Finally, some key improvements to the system are proposed to the design.

D. Amjad M. Abood (2009), Presented paper on A Novel Cam-Based Infinitely Variable Transmission [4]. This paper presents infinitely variable transmission system, is the system which allows for continuous variation of transmission ratio including zero transmission ratio. In this paper, the geometric design and kinematics analysis for a novel cam based infinitely variable transmission system are presented. The proposed system has a number of identical units, each unit contains three dimensional cam follower, grooved wheel, and one way clutch (ratchet). Through each unit the rotational motion is converted to an oscillatory linear motion of variable amplitude and then rectified to rotational motion again. The mathematical expressions for the kinematics analysis are formulated and simulation is established according to this formulation. It was concluded that at fixed transmission ratio, the system produces uniform output for uniform input. On the other hand, during transmission ratio variation there is a little fluctuation in the values of the output angular velocity and acceleration. This fluctuation is decreased when the number of units is increased. Also it was concluded that the maximum transmission ratio of the system depends on the geometric design factors.

E. Giuseppe Carbone (2009) The Dissertation Presented on Modelling, Optimization and verification of power split infinitely variable transmissions [5]. This Dissertation presents the author presents an optimization procedure to design infinitely variable transmission architectures which allows them to achieve a significant reduction of power recirculation and, hence, an increase in mechanical efficiency. The focus of this thesis is on infinitely variable transmissions used in off-highway vehicles and in particular on input coupled and output coupled architectures. The optimized solutions have been analyzed in depth, with particular attention to the power flowing through the infinitely variable unit, which strongly influences the overall efficiency of the transmission. The major result of this study is that also the so far neglected output coupled solution, if properly optimized, guarantees very good performance over the entire range of vehicle speed. The analysis then shows that the particular choice of either input or output coupled architecture by 
itself, or of a mixed solution, strictly depends on the specific application under consideration and that none of them should be discarded a priori.

F. Ranbir Singh (2012) Presented paper on a brief review of transmission in automobiles [6]. In this paper the need for a transmission in an automobile is a consequence of the characteristics of the internal combustion engine. Engines typically operate over a range of 600 to about 7000 revolutions per minute (though this varies, and is typically less for diesel engines), while the automobile vehicle wheels rotate between $0 \mathrm{rpm}$ and around $1800 \mathrm{rpm}$. Furthermore, the engine provides its highest torque outputs approximately in the middle of its range, while often the greatest torque is required when the vehicle is moving from rest or traveling slowly. Therefore, a system that transforms the engine's output so that it can supply high torque at low speeds, but also operate at highway speeds with the motor still operating within its limits, is required.

G. Konstantin Ivanovo (2013) Presented paper on A CAD Design of a New Planetary Gear Transmission [7]. This paper presents the design and characterization of a new planetary gear transmission with two degrees of freedom. The main purpose of the planetary gear transmission is on the capability to adapt the operation to variable loading. Designed adaptation provides a motion of output link with a speed that is inversely proportional a loading of the link. A detailed 3D CAD model has been proposed in order to investigate the operation feasibility of the proposed design solution.

H. F.G. Benitez (2004) Presented paper on infinitely variable transmission of Ratcheting drive type based on one-way clutches [8]. The prior work in Infinitely Variable Transmission System by using Ratcheting drives by a transmission whose characterized by its non-uniform output for a uniform input. It is similar in that there is a device that varies the amount of rotation of several planetary gears with respect to a carrier. This particular design uses a slotted plate to drive a number of planet gears around a sun gear. By varying the eccentricity, e, of the slotted plate with respect to the "guide groove", the angular velocity of the planet gears will vary as they travel around the sun. Each planet gear is connected to a second planet through a one way clutch; the planet with the largest velocity will then transmit motion to the ring gear.

I. P. B. Pires (1991) Presented paper on A geared infinitely variable transmission for automotive applications.[9] This paper presents incorporates a number of levers, shafts, and a slotted plate to accomplish the same task. Similar in concept to both Pires and Benitez, but different in implementation is a design used by Matsumoto (2003) which is commercially available under the name "Zero-Max".[10] This transmission uses severalreciprocating four bar linkages to oscillate the indexing clutches. One of the several linkage systems. An input is applied to the crank mechanism on the left which oscillates the first four bar mechanism. A second four bar loop converts these oscillations to a continuous output rotation through a number of one way clutches. Like Benitez"s and Pires' CVTs though, it exhibits a non-uniform output for a uniform input.

J. Douglas Magyari (1999) Presented Patent on Infinitely Variable Transmission [11]. In this patent the same vein as other ratcheting drives, develop an Infinitely Variable Transmission comprising a pair of rotary, generally conical, torque-transmitting members, each being mounted for rotation on its geometric axis, the angularity of axes, one with respect to the other, being variable, the outer surfaces of each member having torque transmitting needles extending outwardly from the generally conical surface, the needles of one member meshing with the needles of its companion member, the needles being capable of flexing whereby torque may be transmitted through the rotary members without frictional sliding motion at the area of meshing engagement of the needles, the angularity of one member with respect to the other permitting a wide torque transmitting ratio range.

K. Peter Eichenberger (1988) Presented Patent on Dual Range Infinitely Variable Transmission [12]. In this patent improving torque transmitting capacity has worked which having a driving sheave assembly connected to the crankshaft of an internal combustion engine and coaxially mounted with respect to the crankshaft, an intermediate shaft upon which is mounted a driven pulley assembly, a drive chain or belt drivable connected the pulley assemblies, high range and low range gearing coaxially disposed with respect to the driven pulley assembly, a countershaft arranged in parallel and spaced disposition with respect to the secondary shaft wherein the countershaft is adapted to support forward and reverse gearing and improved bearing means for supporting the countershaft and wherein a differential output assembly connects torque output elements of the countershaft to each of two axle half shafts thus providing improved torque transmitting capacity with reduced overall dimensions due to the reduced shaft spacing.

L. Wayne Paul Bishop (2011) Presented Patent on Positive Drive Infinitely Variable Transmission [13]. In this patent Infinitely Variable Transmission by using Forced Way method. This is a unique method of generating variable ratio outputs from a given input of constant rotational speed by forcing one end of a drive shaft to follow a continuous path (way) around a given circle at a constant speed that can then be deformed into curves of varying radiuses that would range from the radius of the given circle to that of the various radiuses (arcs) of various ovals to a final shape of an oblong. Forcing the end of the drive shaft to follow these shapes would result in the driveshaft rotating at different rotational speeds as it transverses the 
shape. By sampling only the rotational output while moving over the desired portion of the shape one can control the outputs rotational speed which will be some ratio of the input.

\section{Theoretical Construction And Working Of IVT:}

The input to the Mechanical Torque Convertor, the motor, produces a power and torque output that is constant with respect to time, at a given speed. This constant power and torque, is transmitted to the arm assembly via the input assembly. The IVT in turn converts the constant input into a sinusoidal, oscillating torque via its specific mechanism; the clutch assembly of the mass-inertia drive converts the oscillating power output from the arm assembly into unidirectional power pulses. The average power is dependent on the amplitude and frequency of these pulses. Higher amplitude and frequency will result in a higher average power output. The amplitude of the power pulses depends on the magnitude of the input received from the engine, while the frequency of the pulses is dependent on the speed of the arm assembly shaft.

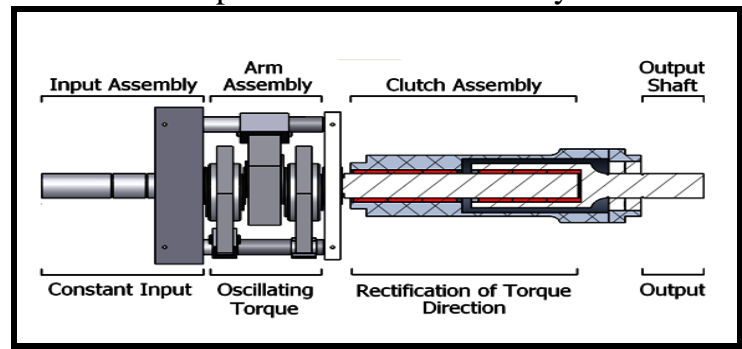

Figure-2. Mechanical Torque Convertor of IVT

The Mechanical Torque Convertor can be considered to consist of four main parts. These are the input assembly, arm assembly, clutch assembly and the output shaft. All of these areas serve a specific purpose in the operation of the mass-inertia drive. The input assembly delivers the input from the engine, the arm assembly generates oscillating torque, the clutch assembly rectifies that oscillating torque to a consistent direction, and the output shaft delivers the output to the rest of the drive train. These sections and their associated functions can be seen in Fig.3

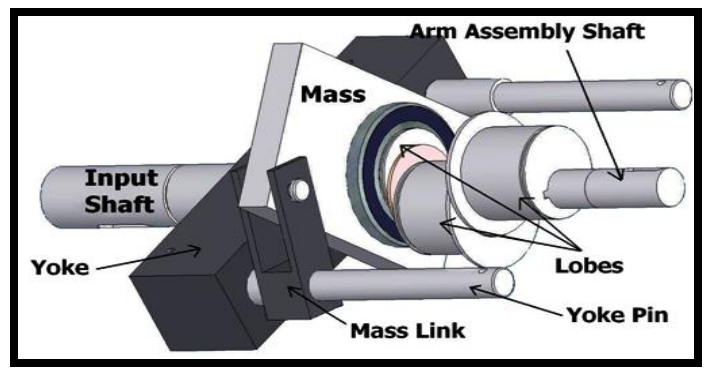

Figure-3. Mechanical Torque Convertor in 3-D view of IVT

The heart of the mass-inertia drive is the method in which the masses interact with the arm assembly. This arm assembly allows for the masses to generate torque through their rotation and transmits it to the output shaft. The central shaft of the arm assembly has three lobes attached to it. These lobes are circular pieces of steel with an offset bore for the arm assembly shaft. The center lobe is fitted with the largest of the three masses, while the outer two lobes are each fitted with a mass half the size of the largest mass.

The maximum torque is given by the formula:

$T=\mathbf{m} * \omega 2 * R_{C G} * D$ (lobe offset)

These four stages demonstrate the oscillatory nature of the torque generated by the mass-inertia drive, This configuration ensures that the shaft is balanced; the two masses on one side of the shaft equal the mass of the largest mass on the opposite side of the shaft.

\section{Conclusions:}

Today world save energy parameters are use. The most of transmission system is develop the energy but west. Now's day linear transmission system IVT is part energy saving in the research and develop as the development continuous fuel efficiency and performance benefit invariability increase this will lead increase such Three masses mechanism. The Three masses profile further development of masses. The liner repeated of the transmission ratio. In any technology with inherent benefits eventually reach fruition the IVT has only just begun to blossom.

\section{Acknowledgement:}


I gratefully acknowledge Mechanical engineering department of Alard COE\&M, Pune for technical support and providing the research facilities. I would also like to thank to Dr. S. B. Padwal, Principal (Alard COE\&M, Pune) and Prof. V. R. Bajaj HOD (Mechanical department) for their help and dedication toward my research and related research, also my friends for their directly \& indirectly help, support and excellent co-operation.

\section{Journal Papers:}

\section{References}

[1] Dr. N. Arunkumar, Infinitely Variable Transmission Using Four Bar Mechanism, International Journal of Engineering Science and Technology (IJEST) 2014; 6(4):170-176

[2] Amjad M. Abood, A Novel Cam-Based Infinitely Variable Transmission, Journal of Kerbala University, 2010; 8(4): 61-74

[3] Ranbir Singh, a brief review of transmission in automobiles, International Journal of Latest Research in Science and Technology, 2012; 1(2):222-226

[4] Konstantin Ivanovo, A CAD Design of a New Planetary Gear Transmission, International Journal of Innovative Technology and Research, 2014; 4(2):1063-1067.

[5] F.G. Benitez, J.M. Madrigal, J.M. del Castillo, Infinitely variable transmission of Ratcheting drive type based on one-way clutches, Journal of Mechanical Design, July 2004; 126:.673-682.

[6] F.A. Fitz, P.B. Pires, A geared infinitely variable transmission for automotive applications, SAE paper no. 910407, 1991:1-7

[7] S. Matsumoto, N. Inoue, Y. Tsukada, Continuously variable transmission for Bicycles, United States Patent Application 20030221892, 2003.

[8] Douglas Magyari, Infinitely Variable Transmission, Patent Application Publication, on 01 November, 1999.

[9] Peter Eichenberger, Dual Range Infinitely Variable Transmission, Patent Application Publication, on 07 July, 1988.

[10] Wayne Paul Bishop, Positive Drive Infinitely Variable Transmission, Patent Application Publication, 04 August, 2011.

[11] Lohr, Charles, Stevenson, Gregory, Infinitely Variable Transmissions, Methods, Assemblies, Subassemblies and Components, Patent Application Publication, 10 December, 2009.

[12] Dieter Hahne, Infinitely Variable Transmission for Automotive Vehicle Driveline, Patent Application Publication, on 21 February, 1984.

[13] Paul K. Coronel, Dual Concentric Positively Infinitely Variable Rotary Motion Transmission, Patent Application Publication, on 16 November, 1992.

\section{Theses:}

[14] Giuseppe Carbone, Modelling, Optimization and verification of power split infinitely variable transmissions, Ph.D. Dissertation, Northwestern University, 2009.

[15] Brian S. Andersen, An Investigation of a Positive Engagement Continuously Variable Transmission, Dissertation at Brigham Young University, Harry Valentine, August 2005.

[16] E. Faulring, The cobotic hand controller: Design, control and analysis of a novel haptic display, Ph.D. Dissertation, Northwe stern University, 2005.

\section{Proceedings Papers:}

[17] Derek F. Lahr, Dennis W. Hong, The Operation And Kinematic Analysis Of A Novel Cam-Based Infinitely Variable Transmission, ASME 2006 International Design Engineering Technical Conferences \& Computers and Information in Engineering Conference September 10-13, 2006, Philadelphia, Pennsylvania, USA.

[18] Gregory F. Hickman, Kinematic Modeling and Analysis of a Cam Based CVT for a Capstone Design Project Experience, Proceedings of the 2013 ASEE North-Central Section Conference, American Society for Engineering Education.

\section{AUTHOR's BIOGRAPHY:}

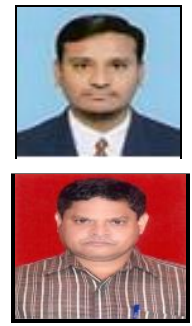

Arun M. Meshram, Currently pursuing M.E in Design Engineering (Mechanical Engineering) Department of Mechanical Engineering From Alard College of Engineering, Pune (India)

Email: arunmeshram82@gmail.com

S. P. Chaphalkar, Currently working as Head of Department, Department of Automobile Engineering at Pimpri Chinchwad, Polytechnic, Pune (India)

Email: chaphalkar77@gmail.com

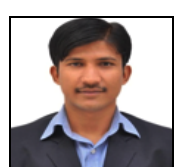

S. N. Khetre, Currently working as an Assistant Professor, Department of Mechanical Engineering at Rajarshi Shahu College of Engineering, Tathawade, Pune (India)

Email: subhash2010khetre@gmail.com

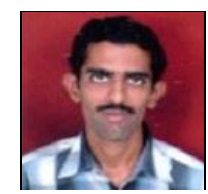

S. R. Wankhede, Currently working as an Assistant Professor, Department of Mechanical Engineering at Pimpri Chinchwad College of Engineering, Pune (India)

Email: sagar.wankhede2007@rediffmail.com 